\title{
Motion and position shifts induced by the double-drift stimulus are unaffected by attentional load
}

\author{
Harry H. Haladjian ${ }^{1} \cdot$ Matteo Lisi $^{2}$ • Patrick Cavanagh ${ }^{1,3,4}$
}

Published online: 23 February 2018

(C) The Psychonomic Society, Inc. 2018

\begin{abstract}
The double-drift stimulus produces a strong shift in apparent motion direction that generates large errors of perceived position. In this study, we tested the effect of attentional load on the perceptual estimates of motion direction and position for double-drift stimuli. In each trial, four objects appeared, one in each quadrant of a large screen, and they moved upward or downward on an angled trajectory. The target object whose direction or position was to be judged was either cued with a small arrow prior to object motion (low attentional load condition) or cued after the objects stopped moving and disappeared (high attentional load condition). In Experiment 1, these objects appeared $10^{\circ}$ from the central fixation, and participants reported the perceived direction of the target's trajectory after the stimulus disappeared by adjusting the direction of an arrow at the center of the response screen. In Experiment 2, the four double-drift objects could appear between $6^{\circ}$ and $14^{\circ}$ from the central fixation, and participants reported the location of the target object after its disappearance by moving the position of a small circle on the response screen. The errors in direction and position judgments showed little effect of the attentional manipulation - similar errors were seen in both experiments whether or not the participant knew which double-drift object would be tested. This suggests that orienting endogenous attention (i.e., by only attending to one object in the precued trials) does not interact with the strength of the motion or position shifts for the double-drift stimulus.
\end{abstract}

Keywords attention $\cdot$ spatial localization $\cdot$ visual perception

The double-drift stimulus produces a strong shift in motion direction and results in large errors of perceived position. This effect occurs when an object moves in the periphery of the visual field in one direction while having a surface texture that moves in the orthogonal direction. The perceived trajectory can deviate by $50^{\circ}$ or more from its actual path, suggesting that the two motion vectors (external and internal) are combined (Shapiro, Lu, Huang, Knight, \& Ennis, 2010; Tse \& Hsieh, 2006).

Harry H. Haladjian

haroutioun@gmail.com

1 Laboratoire Psychologie de la Percetion, CNRS (UMR 8242), Universite Paris Descartes, Sorbonne Paris Cite, Paris, France

2 Centre for Applied Vision Research, City, University of London, London, UK

3 Psychological and Brain Science, Darmouth College, Hanover, NH, USA

4 Department of Psychology, Glendon College, Toronto, ON, Canada
Despite this strong perceptual effect, eye movements made toward the stimulus go to the actual, not the perceived, location (Lisi \& Cavanagh, 2015). Since the oculomotor system is immune to this large perceptual effect, it suggests that visual attention, which is strongly linked to the oculomotor system (e.g., see Awh, Armstrong, \& Moore, 2006; Deubel \& Schneider, 1996; Rizzolatti, Riggio, Dascola, \& Umiltà, 1987; Casarotti, Lisi, Umiltà, \& Zorzi, 2012), may be as well.

In the current study, we examined the effect of attentional load on the double-drift illusion to determine whether increasing the demands on attention will affect the integration of the two motion sources that produces this illusion. We manipulated attentional load and asked participants to estimate the direction of target object motion (Experiment 1) or to indicate the perceived final target location (Experiment 2). In both experiments, four double-drift objects were presented, one in each quadrant of a large screen, and they moved upward or downward along an oblique trajectory. After $400 \mathrm{~ms}$, the objects disappeared and a pointer indicated the quadrant of the 
target to be reported. Participants responded with either the direction or location of the object that had been in the target quadrant. Two conditions were used to manipulate attentional load. In the first condition, one of the quadrants was precued as the target with a small arrow at the beginning of the trial, and the double-drift object in that quadrant was always the one tested at the end of the motion. Participants could then focus attention on that target and ignore the others (the low attentional load, or Track 1 condition). In the other attention condition, a neutral cue was given at the start so that participants had to attend to all four objects, and the target quadrant was cued only after the stimulus disappeared at the end of the trial (the high attentional load, or Track 4 condition). In this high attentional load condition, we assume that attention is distributed over the four stimuli, and less is available for the target that will be probed.

Tracking multiple objects is an attention-demanding task (Drew, McCollough, Horowitz, \& Vogel, 2009) with a limited capacity for the number of objects that can be tracked simultaneously. This limit is usually found to be around four objects, but it can fluctuate depending on tracking conditions (e.g., see Alvarez \& Franconeri, 2007; Cavanagh \& Alvarez, 2005; Pylyshyn, 1989). Typical experiments using this paradigm find that the positions of four objects can be tracked with few errors, but object features, including predicted trajectories, are not encoded accurately (Keane \& Pylyshyn, 2006; Pylyshyn, 2004). When tracking only one or two objects, such features can be encoded and allow for predictions of motion trajectories (Fencsik, Klieger, \& Horowitz, 2007; Horowitz \& Cohen, 2010). By manipulating the number of objects to be tracked in our experiment, we manipulated the availability of attentional resources (e.g., see Drew et al., 2009; Lisi, Bonato, \& Zorzi, 2015; Meyerhoff, Papenmeier, \& Huff, 2017), which in turn can assist in position and trajectory estimates. If focused attention is responsible for the integration of motion signals that produces the double-drift illusion, then we would expect a reduced effect of the illusion when attentional load is high (i.e., a smaller magnitude of error). If there are no direction or position judgment differences between low and high attention load conditions, but still an effect of the illusion, then it is possible that the attention used for focused tracking is not involved in the integration of motion signals in the double-drift illusion, and that this integration occurs earlier in the processing stream and not in the systems used for programming eye movements (Lisi \& Cavanagh, 2015). Alternatively, if there are smaller direction or position judgment errors under low attentional load, it may be possible that focused attention reduces the strength of the illusion and improves the precision in performing the experimental tasks.

\section{Experiment 1: Judging direction of double-drift object motion}

In this experiment, participants were asked to report the direction of a moving double-drift object under low and high attentional load conditions.

\section{Participants}

Eight participants were recruited from the Université Paris Descartes. All participants (except two authors) were paid 10 euros for 1 hour of participation. Informed consent was provided by the participants under a research protocol approved by the Universite Paris Descartes Review Board, CERES, in accordance with French regulations and the Declaration of Helsinki.

\section{Stimuli}

Each trial presented four circular objects that were $\sim 1^{\circ}$ (degree visual angle), with one object in each quadrant of a gray screen at a distance of $10^{\circ}$ from the central fixation. These double-drift objects consisted of $1 / \mathrm{f}$ luminance noise presented within a Gaussian contrast envelope with $\sigma=$ $0.2^{\circ}$, and moved at $10^{\circ}$ per second along a direction randomly sampled from a uniform distribution of all directions $\left(0^{\circ}-360^{\circ}\right.$, in increments of $\left.10^{\circ}\right)$; the motion trajectory was $4^{\circ}$ in length. The internal noise pattern drifted in a direction orthogonal to the movement of the envelope (either $+90^{\circ}$ or $-90^{\circ}$, intermixed presentation) with a speed of $6^{\circ}$ per second. Since the orientation of the motion trajectory was randomized for each object in each trial, the starting and end points of the objects were not the same across trials, reducing any potential strategies the observers could make for estimating the direction of movement.

\section{Target cueing}

The cue indicating which object had to be tracked was provided either before the stimulus presentation (low attention, Track 1 condition), allowing participants to focus attention on one target object, or after the presentation (high attention, Track 4 condition), requiring participants to split attention among all four moving objects. The cue consisted of a short black line ( $1.25^{\circ}$ in length), starting at fixation and pointing to the quadrant of the target; in the postcue condition, the presentation of the stimulus is preceded by a neutral cue, which consisted of four short lines pointing to all four quadrants, with the postcue appearing after the disappearance of the stimuli. 


\section{Response method}

A small arrow (2.5 in length) pointing toward a random orientation appeared at the center of screen after the postcue, and the participant moved the mouse to adjust the direction of the arrow to best match the perceived motion direction of the target object. There was a $200-\mathrm{ms}$ interstimulus interval (ISI) between postcue offset and the arrow response tool appearance. Participants clicked the mouse when they were satisfied with their direction response, which then initiated the next trial.

\section{Experimental conditions}

This experiment manipulated attentional load with Track 1 or Track 4 trials. These conditions were randomly intermixed within one experimental session of 576 trials. These trials were split into six blocks lasting approximately 5-7 minutes each, allowing for a break in between blocks; the participant was recalibrated on the eye tracker before a block that followed a break.

\section{Apparatus}

The experiment was programmed in MATLAB 2014b using the Psychophysics Toolbox (Brainard, 1997), and testing was completed on an Apple Mac Pro desktop computer (Quad-Core Intel Xeon $2.8 \mathrm{GHz}$, running OSX 10.6.3) with an NVIDIA GeForce 8800 GT graphics card. Stimuli were presented using a PROPixx DLP color projector system (VPX-PRO-5001c; VPixx Technologies, Inc., Saint-Bruno, Canada) at a resolution of $1920 \times$ 1080 pixels and a refresh rate of $120 \mathrm{~Hz}$; the screen's viewing area was approximately $65^{\circ} \times 37^{\circ}(137.5 \times 78$ $\mathrm{cm})$. Eye movements were monitored with an SR Research EyeLink 1000 desktop eye tracker (controlled by a dedicated computer), which tracked the right eye at $1000 \mathrm{~Hz}$.

\section{Procedure}

Participants were seated in front of the screen at a fixed distance of $120 \mathrm{~cm}$; a chin rest was used to limit head movements. The eye tracker was calibrated on the participant at the start of the session. Before the first test block, 10 practice trials were completed to familiarize the participant with the task. The experimenter instructed the participant to fixate at the center of the screen on a small dot $\left(0.25^{\circ}\right.$ in diameter $)$ in order to initiate the trial. After 400 $600 \mathrm{~ms}$ of proper fixation (i.e., within $2.5^{\circ}$ of this dot), the quadrants with the targets to be tracked were cued for $1,000 \mathrm{~ms}$ (i.e., either one or four objects to be tracked, intermixed within the session). Next, the four double-drift objects appeared in the quadrants and moved simultaneously along the designated trajectory in each quadrant; this motion lasted $400 \mathrm{~ms}$. Once the movement was completed, the objects disappeared, and after a 200-ms ISI, a cue appeared in the postcue condition, indicating which one of the four objects had to be reported. A small arrow at the center of the screen then appeared and the participant used the computer mouse to report the perceived motion direction of the target object. The next trial began after a $1000-\mathrm{ms}$ blank screen that appeared after the response was made. Gaze had to be maintained within $2.5^{\circ}$ of the central fixation during the precue and stimulus presentation, or else the trial was aborted and a message appeared reminding the participant to keep their gaze at the central fixation; aborted trials were repeated at the end of the block (see Fig. 1 for a schematic of a trial).

\section{Analyses}

The responses were analyzed by determining the difference between the real direction of the movement of the object and the reported direction. These deviations (i.e., errors) were transformed so that positive values indicated that the reported directions were shifted from the real direction toward the direction of the internal motion. The mean error standard deviation was computed for each participant for each tracking condition, and trials with errors greater than three standard deviations from the mean were excluded from the analyses, or $4.7 \%$ of trials.

Two attentional load conditions were compared in these experiments by varying the number of objects to be tracked in order to manipulate the availability of attentional resources (see Drew et al., 2009). Attentional load could, in principle, influence the responses in several ways: (1) change the bias (i.e., the mean error), (2) decrease the precision of the responses (i.e., increase the standard deviation), and (3) eventually increase the proportion of random guesses. Therefore, we analyzed the responses using a probability model that allowed us to disentangle these three aspects: The model consists of a mixture between a von Mises (similar to a Gaussian distribution but wrapped around the circle) and a uniform distribution for the direction responses. The uniform distribution allows the model to represent the probability that participants randomly guessed the orientation of the target in trials where they did not see it (e.g., because of attentional lapses). See the Appendix for model formulas.

To examine overall effects of the attentional manipulation, a mixed-model analysis of variance (ANOVA) was conducted on data from all trials and included the participant (ID) as a random variable in the model to account for both within-subject and between-subject variability. 


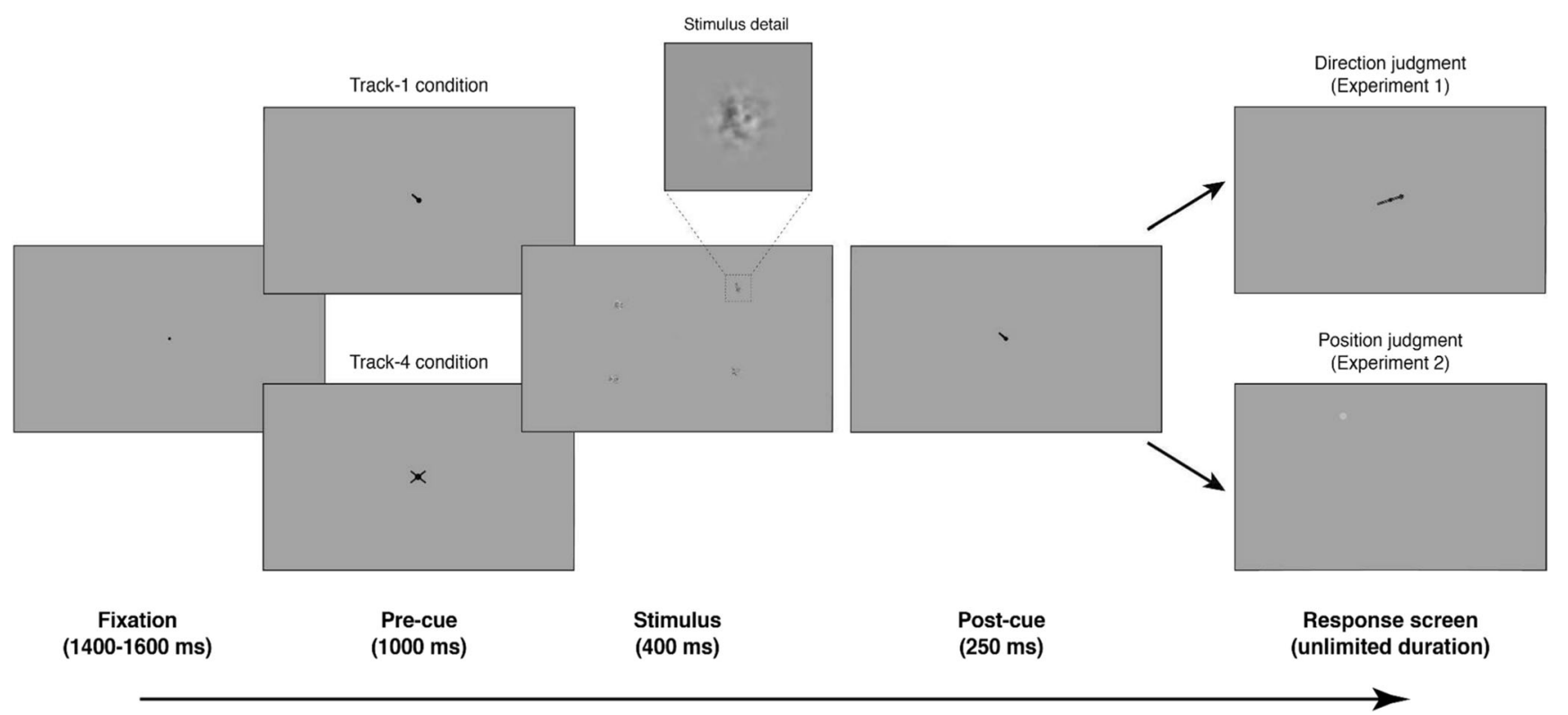

Fig. 1 Schematic of trial. After eye-tracker confirmed gaze at central fixation, the pre-cue screen appeared and indicated the quadrant with the target that was to be tracked (Track 1 condition) or whether all quadrants would need to be tracked (Track 4). The stimulus then appeared and moved along the designated trajectory in one motion sweep (i.e., no reversal). The postcue then appeared either confirming the precue or indicating which of the four tracked objects was to be

This keeps the within-subject variability in responses that would otherwise be overlooked when taking the average performance of each condition for each participant (e.g., in repeated-measures analyses). The key results reported reported. After a short blank, the response screen appeared: In Experiment 1, it was a central arrow whose direction could be changed by moving the mouse to indicate perceived direction of motion; in Experiment 2, a small gray circle appeared, which could be adjusted along an invisible line to indicate the perceived final position of the target object. Note. Dimensions of stimuli and screen are not drawn to scale

are analyses on pooled data, with individual-level results shown to illustrate the variability in performance among the participants.

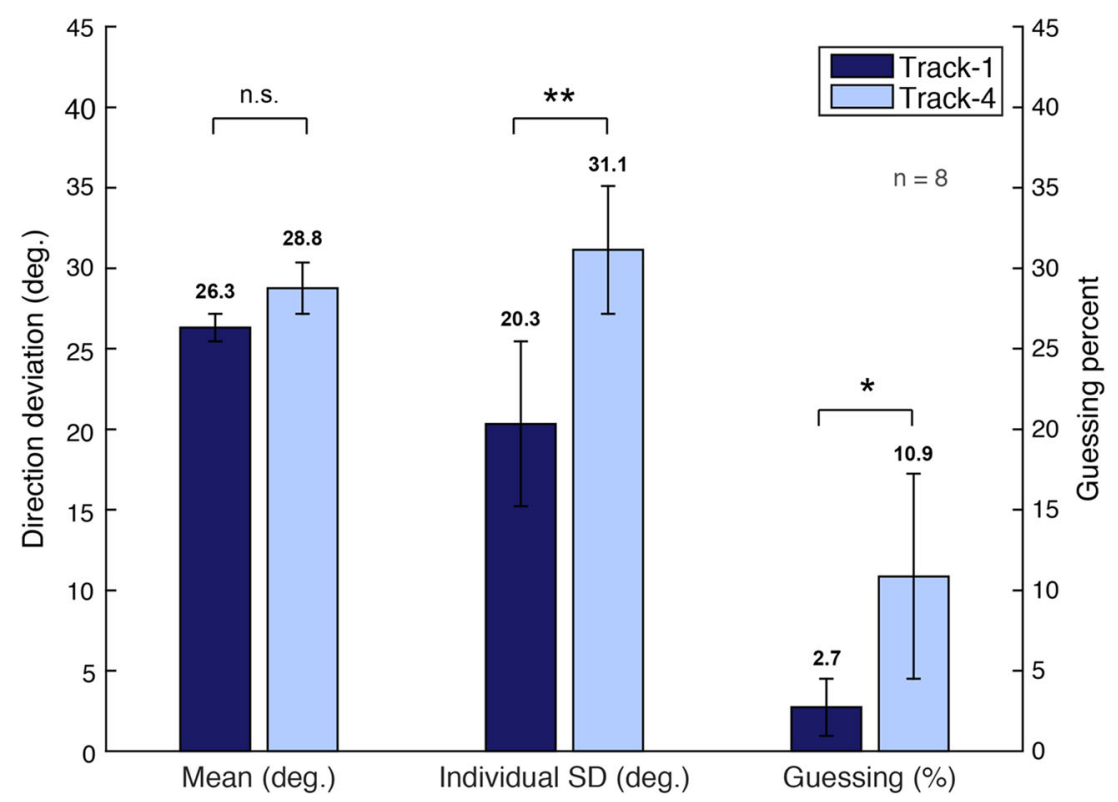

Fig. 2 Left: Deviation of responses in perceived direction of double-drift stimulus from true direction for Track 1 (low load) and Track 4 (high load) conditions. Mean of the standard deviations of the participants' direction responses is plotted in the middle and the guessing rates, extracted from a mixed model of the task performance, is shown on the right (right hand vertical axis). Note. Error bars indicate $95 \%$ confidence intervals. $* p<.01 . * * p<.001$ 


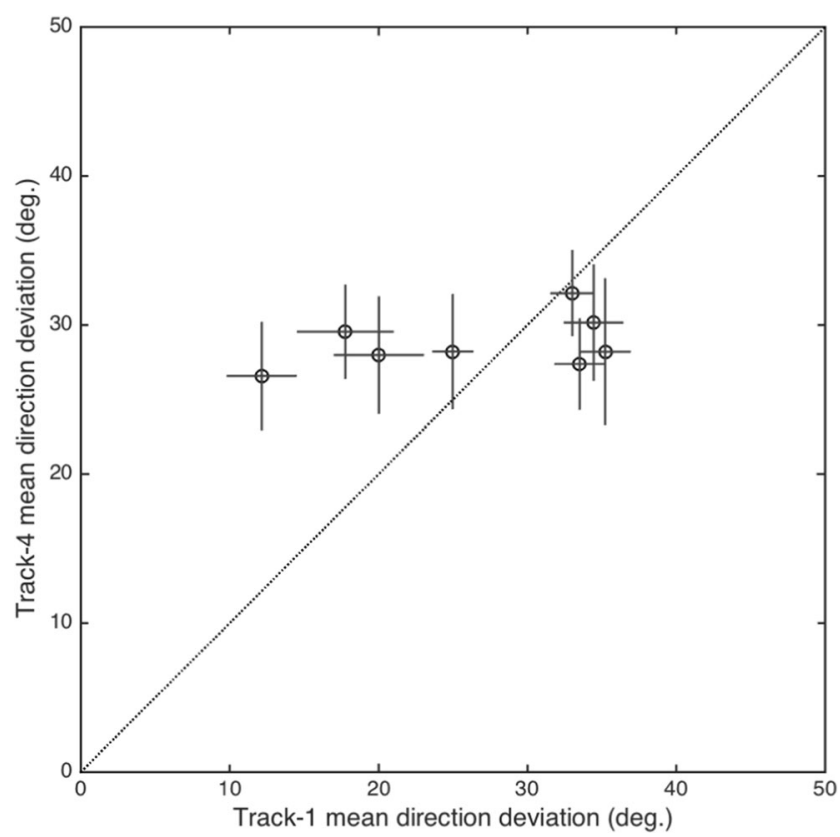

Fig. 3 Perceived direction deviation from true direction for the Track 4 condition plotted against the deviation for the Track 1 condition, for each participant individually $(n=8)$. Error bars indicate $95 \%$ confidence intervals

\section{Results}

Overall, there was a significant deviation in the perceived orientation of the target's motion path in both the Track 4 and Track 1 conditions $\left(28.8^{\circ} \pm 0.66,26.3^{\circ} \pm 0.44\right.$, respectively, both $p$ 's $<.01$; see Fig. 2). The difference between the two attention conditions, however, was not significant, $F(1,4376)=0.65, p=.45$. Response variability did increase in the Track 4 condition, Brown-Forsythe test for equal variances: $F(1,4390)=150.82, p<.001$, with all but two of the participants having this trend in variability $(p s<.01)$. This suggests an effect of the attentional load on the task, even if there was no effect on the illusion strength. Similarly, a mixture model of the results (see Table 1 in the Appendix) allowed an estimate of the proportion of trials with a guessing response, and here, too, there was a significant increase for the Track 4 condition compared to the Track 1 condition, $F(1,14)=$ $12.21, p=.004$, indicating that participants had no information about the target on a larger proportion of Track 4 trials. When they did have information, however, it was similar to that from the Track 1 condition in terms of direction.

The effect of attentional load was variable when tested individually: three of the participants showed no difference, three showed significantly larger direction shifts in the Track 4 condition, and two showed larger shifts in the Track 1 condition. Figure 3 plots the illusion strength in the high attentional load against its strength in the low- load condition for each participant (see Table 1 in the Appendix for individual-level results showing these differences in performance).

There is the possibility that some participants did not focus attention on only one object in the low attentional load condition where the target item was identified in advance. Perhaps the directions of all four targets form a Gestalt pattern that could be attended as a single configuration making the judgment for the one target among four as easily accessible as the individually tracked target. The directions of the four objects were randomized to avoid any obvious symmetry or other cues that would help any grouping, but it is possible, even if unlikely, that the directions of the four items could be perceived as a group as well as the direction of any one of them. To ensure that all four items were tracked individually in the Track 4 condition, we conducted a second experiment that asked participants to judge the final location of the target.

\section{Experiment 2: Judging position of double-drift objects}

Experiment 2 used the same stimuli as in Experiment 1, but instead of reporting motion direction, participants estimated the final location of the target double-drift stimulus by clicking on the screen with a mouse. We assumed that an accurate estimate of offset position would require more focal attention to the individual target than was perhaps required for the direction judgment. We also manipulated the distance that the objects appeared from the central fixation in order to randomize the final landing position of the target and examine eccentricity effects.

\section{Participants}

Eight participants were recruited from the Universite Paris Descartes, three of whom had also participated in Experiment 1. All but one of the participants (one of the authors) were paid 20 Euros for completing two 1-hour sessions. Informed consent was provided by the participants under a research protocol approved by the Université Paris Descartes Review Board, CERES, in accordance with French regulations and the Declaration of Helsinki.

\section{Stimuli}

Each trial presented four circular objects that were $\sim 1^{\circ}$ in diameter, with one object in each quadrant of a gray screen at a distance of $6^{\circ}, 8^{\circ}, 10^{\circ}, 12^{\circ}$, or $14^{\circ}$ from the central fixation; all four objects were the same distance 
from the central fixation. These double-drift objects were identical to those used in Experiment 1 (with the same speed of movement), but moved up or down along a path that was only $+45^{\circ}$ or $-45^{\circ}$ from vertical, with a slightly higher speed of the internal noise pattern, which drifted at a speed of $8^{\circ}$ per second in a direction orthogonal to the movement of the envelope (either $+90^{\circ}$ or $-90^{\circ}$, intermixed presentation). Since the direction of the motion trajectory and the distance from fixation were randomized for each object in each trial, and the orientations of the nontarget object trajectories were randomized, observers could not develop any strategies for judging the end point of the target object, because this was inconsistent over trials.

\section{Target cueing}

The cue indicating which object would be probed was presented in the same manner as in Experiment 1.

\section{Response method}

After the offset of the postcue (200-ms ISI), a small gray dot appeared at the inner or outer end of an invisible line (402-500 pixels in length) that was placed orthogonal to the trajectory of the target object and which passed through the end point of the stimulus trajectory. The participant moved the dot along this invisible line to indicate the perceived location of the target's disappearance. The response was limited along this line to reduce the noise in responses.

\section{Experimental conditions}

In this experiment, two variables were manipulated: attentional load (Track 1 vs. Track 4) and the eccentricity of the four objects from the central fixation $\left(6^{\circ}, 8^{\circ}, 10^{\circ}, 12^{\circ}\right.$, or $\left.14^{\circ}\right)$. Each participant completed two sessions, with 480 trials per session. The conditions were randomly intermixed among the 480 trials and split into three blocks lasting approximately 15 minutes each, allowing for a break in between blocks; the participant was recalibrated on the eye tracker before each block.

\section{Apparatus}

The experimental equipment (i.e., computers, projector, and eye tracker) was the same as in Experiment 1.

\section{Procedure}

Participants were seated in front of the screen at a fixed distance of $120 \mathrm{~cm}$; a chin rest was used to limit head movements. The eye tracker was calibrated for each participant at the start of the session. Before the first test block, 10 practice trials were completed to familiarize the participant with the task. The experimenter instructed the participant to fixate the center of the screen on a small dot $\left(0.25^{\circ}\right.$ in diameter $)$ in order to initiate the trial. After 400-600 ms of fixation (maintained within $2.5^{\circ}$ of this dot), the quadrants that would contain the targets to be tracked were cued for $1,000 \mathrm{~ms}$ (i.e., either one or four objects to be tracked). Next, the four double-drift objects appeared in the quadrants and moved simultaneously along the designated trajectory in each quadrant; this motion lasted $400 \mathrm{~ms}$. Once the movement was finished, the objects disappeared and after a 200-ms ISI, a 250-ms cue appeared either confirming the single object being tracked (the precue condition) or indicating which one of the four objects being tracked had to be localized (the postcue condition). Then a gray circle appeared in the target quadrant and the participant used the computer mouse to slide the circle along an invisible line to the location that best matched the perceived final location of the target object. The next trial began after a 1,000-ms blank screen that appeared after the response was made. Gaze had to be maintained within $2.5^{\circ}$ of the central fixation during the precue and stimulus presentation or else the trial was aborted and a message appeared reminding the participant to keep their gaze at the central fixation; aborted trials were repeated at the end of the block.

\section{Analyses}

The responses were analyzed by determining the distance between the participant's response and the actual location of the target object, which was computed as the degrees of rotation from the physical path (to be comparable to Experiment 1 errors). For each trial, this distance measure was attributed a positive or negative sign such that positive values indicate an error in the direction of the internal motion (i.e., double drift) and negative values indicate an error opposite the direction of the internal motion. The mean position error and the standard deviation were computed for each participant for each condition (tracking and eccentricity), and trials with errors greater than three standard deviations from the mean were excluded from the analyses, or $7.5 \%$ of trials. Here again we used a mixed-model ANOVA with the participant as a random variable to account for both within-subject and between-subject variability.

\section{Results}

Overall, there was no significant effect of the attentional manipulation across participants-position deviations in the direction of the illusion were similar in both low and high attentional load. The ANOVA results indicated a significant effect of eccentricity, $F(4,7055)=53.13, p<$ 


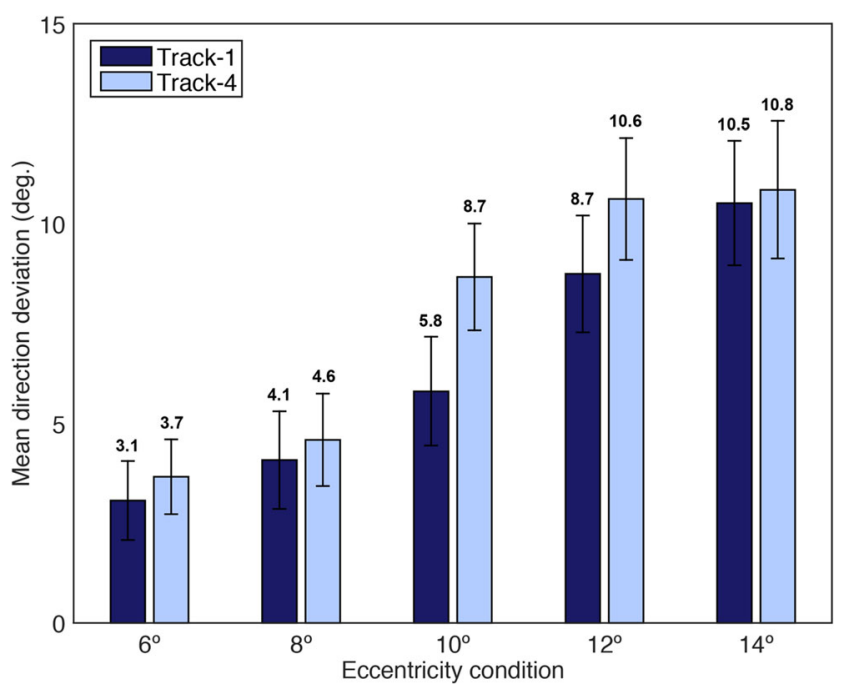

Fig. 4 Average localization errors in Experiment 2 as the signed angle of deviation from true final position, for each eccentricity condition (all participants combined, $n=8$ ); positive values are in the direction of DD. There were no significant differences between Track 1 and Track 4 conditions at any eccentricity

.001 , but no effect of Track 1 versus Track 4 conditions, $F(1,7055)=3.76, p=.09$, and no interaction, $F(4,7055)$ $=1.32, p=.26$. This effect of eccentricity produced an increasing magnitude of position deviation with larger eccentricities and was significant for all participants. Again, the direction of internal motion (i.e., inward vs. outward) did not produce any significant differences in performance. Post hoc analyses on the different tracking conditions found no significant differences in overall performance within the different eccentricities, with only one participant showing a significant difference between the Track 1 and Track 4 conditions at the $6^{\circ}$ eccentricity (see the summary in Table 2 in the Appendix). See Fig. 4 for an overall summary of the results by eccentricity condition. Note that for comparison with Experiment 1, the results from the $10^{\circ}$ eccentricity condition are most relevant (which were not significant).

There appears to be a slightly larger effect of the double-drift illusion when tracking four objects versus tracking one object, but this is not significant overall. The attentional load did not have a significant effect for any participant individually (see Table 2 in the Appendix for a summary of these results). Differences in response variability among the different conditions were analyzed using the Brown-Forsythe test for equal variances. The variability in responses increased with eccentricity, $F(4$, $7102)=114.17, p<.001$, which was significant for each participant $(p \mathrm{~s}<.01)$. There was no significant difference in response variability between the two tracking conditions, $F(1,7105)=1.19, p=.28$.

The effect of the double-drift illusion was weaker in Experiment 2 than in Experiment 1 despite the higher internal motion speed. In Experiment 1, the overall direction judgment deviation was $26.3^{\circ}$ and $28.8^{\circ}$ for the Track 1 and Track 4 conditions, respectively, while in Experiment 2, the equivalent overall angle deviation corresponding to the position errors was $8.2^{\circ}$ and $10.5^{\circ}$, respectively (for the $10^{\circ}$ eccentricity that matched that of Experiment 1). This means that Experiment 2 produced only $31 \%$ to $36 \%$ of the illusion strength that was present in Experiment 1. This suggests that position judgments that are based on the instantaneous, last-seen location might be determined differently than judgments of perceived direction of motion that depend on a longer time interval.

\section{General discussion}

The overall results for direction and position judgments indicate that there was little consistent change in the motioninduced shifts between the two attentional load conditions in both experiments. In Experiment 1, an increase in the guessing rate in the Track 4 condition indicated that there was indeed less attention allocated to the target trajectory without the precue - the participants responded randomly more frequently in this condition. This was also seen in the increased variability of responses. In Experiment 2, the illusion strength as measured by the position shifts was smaller than in Experiment 1 and again was unaffected by the attentional load. The illusory position shifts increased with the increasing eccentricity of the test path. There was no difference in the response variability for the two attention conditions. The absence of an effect of attentional load suggests that the integration of motion information from the two sources (i.e., internal motion and trajectory) can occur without a focused, resourceintensive form of attention, resulting in the position and direction shifts normally seen in the double-drift stimulus with full attention. While there might be some improvement in the precision of responses in the direction judgment task for the low attentional load condition (Experiment 1), there was no strong effect of the low attentional load condition that would indicate that having more attentional resources would improve overall accuracy of the direction or position judgments. This suggests that, to a large extent, the Gabor's offset drew enough attention to itself to support similarly precise judgments independently of the amount of attention allocated to the trajectory that preceded the offset.

A new finding in this study is that the strength of the illusion is stronger when judging direction (Experiment 1) than position (Experiment 2). The overall direction judgment error was $26.3^{\circ}$ and $28.8^{\circ}$ for the Track 1 and Track 4 conditions, respectively, while in Experiment 2, the equivalent overall angle deviation derived from the position errors was $8.2^{\circ}$ and $10.5^{\circ}$, respectively. A recent study using the double-drift stimulus found that using one's finger to point to a target's location also showed less deviation than direction judgments 
(Lisi \& Cavanagh, 2017). Both of these results could be due to having different spatial representations for object direction and object location.

One interpretation of how different spatial representations might result in the performance discrepancy between perceived motion direction and perceived position is that the different representations may rely on the frequency of how often the information is updated, with position information being updated - and any accumulating errors "reset"-more often than direction information. Motion direction judgments may require information to be integrated over a longer period in order to make an adequate decision. It may be that the information from the tracking mechanism is updated more frequently for the position representation while this information is accumulated over a longer time interval for judging the direction of motion, since the direction of a moving object requires more integrated information about past location (resulting in the stronger double-drift illusion for the direction task in Experiment 1). The idea of "resetting" the representation of a moving object's position can account for why position errors do not increase indefinitely when viewing the double-drift stimulus (Kwon, Tadin, \& Knill, 2015), and why the perception of motion is a straight line and not curved (see Lisi \& Cavanagh, 2015). Yet it is unlikely that the perception of motion and position are truly isolated, as they likely rely on the same object-tracking mechanism (see Kwon et al., 2015).

Our evidence for the effectiveness of our attentional manipulation comes from the increased response variability, which is associated with more guessing, in the Track 4 condition in Experiment 1 . The $\sim 27^{\circ}$ effect we found in Experiment 1 is about half of the effect seen with optimal conditions in previous experiments (about 50 $0^{\circ}$ in Lisi \& Cavanagh, 2015; and about $56^{\circ}$ in Kwon et al., 2015). Given this midway effect in our current study, we avoid floor and ceiling issues and should be sensitive to both increases and decreases in the effect. Our results do indicate that if attention does have an effect on the type of motion integration that occurs during the double-drift illusion, it would be a small effect.

\section{Conclusions}

The double-drift illusion results from the integration of two motion signals that come from the movement of an object along its trajectory and the orthogonal internal motion of the moving object. By manipulating the amount of attention available to track these objects, we examined whether focal attention is critical for this integration step. We found that having more attentional resources available for tracking has little effect on the magnitude of errors. Tracking just one object in the Track 1 condition reduces some variability in responses in comparison to the Track 4 condition, but provides an insignificant change in the direction and position shifts of the double-drift effect.

These results indicate that increasing attentional resources has little overall effect on direction or position judgments of the double-drift stimulus, but it can significantly reduce the rate of guessing and response variability under some conditions. Additionally, it appears that different spatial representations may be used for motion judgments and for position judgments. These findings have implications for our understanding of where and how motion information is integrated to support different forms of perceptual judgments for moving objects.

Acknowledgements This research was funded by the European Research Council under the European Union's Seventh Framework Programme (FP7/2007-2013)/ERC grant agreement No. AG324070 awarded to Patrick Cavanagh and support from the Department of Psychological and Brain Sciences of Dartmouth College.

\section{Appendix}

Mixture model description

The probability model used to analyze responses in Experiment 1 had the form

$p(x)=p_{\text {guess }} \frac{1}{2 \pi}+\left(1-p_{\text {guess }}\right) \frac{1}{2 \pi I o(k)} e^{k \cos (x-\mu)}$

where $k$ is a concentration parameter (can be converted into a standard deviation), $x$ is the error (in radians) over which the density is calculated, $\mu$ is the mean direction of the von Mises distribution (average error), and $p_{\text {guess }}$ is the probability of guesses. The parameters $k$ and $\mu$ map closely to those of the mean vector (mean length and direction). The function $I_{0}(x)$ is a modified Bessel Function of the order zero. The general definition for the Bessel function is given by

$I_{n}(z)=\frac{1}{2 \pi} \int_{o}^{2 \pi} e^{z \cos \phi}(n \phi) d \phi$

For the zero order the term $\cos (n \phi) d \phi$ reduces to $\cos 0=1$ and so the equation for $I_{0}(z)$ is reduced to

$I_{o}(z)=\frac{1}{2 \pi} \int_{o}^{2 \pi} e^{z \cos \phi} d \phi$

The parameter $k$ can be converted into a standard deviation with the following formula:

$s d=\sqrt{-2 \log \left(\frac{I_{1}(k)}{I_{0}(k)}\right)}$

All the parameters were fitted on individual data using maximum likelihood estimation. 
Table 1 Mean error and estimated parameters of mixture models for Experiment 1 results (all angles reported in degrees); the last two columns indicate the average bias for each participant

\begin{tabular}{|c|c|c|c|c|c|c|c|}
\hline ID & Condition & Mean error & Standard deviation & $p_{\text {guess }}$ & $S E_{p}$ & $\mu$ & $S E \mu$ \\
\hline 101 & Track 1 & $20.01 *$ & 25.19 & 0.05 & 0.02 & 21.77 & 1.48 \\
\hline 101 & Track 4 & $27.99 *$ & 32.84 & 0.14 & 0.03 & 32.12 & 1.39 \\
\hline 102 & Track 1 & 24.98 & $9.40 *$ & 0.00 & 0.06 & 31.31 & 0.77 \\
\hline 102 & Track 4 & 28.23 & $32.52 *$ & 0.11 & 0.03 & 32.96 & 1.52 \\
\hline 103 & Track 1 & 34.44 & $16.38 *$ & 0.04 & 0.01 & 35.05 & 1.01 \\
\hline 103 & Track 4 & 30.16 & $32.52 *$ & 0.13 & 0.03 & 34.16 & 1.62 \\
\hline 104 & Track 1 & $33.50 *$ & $13.77 *$ & 0.02 & 0.01 & 34.29 & 0.86 \\
\hline 104 & Track 4 & $27.38^{*}$ & $25.46^{*}$ & 0.07 & 0.02 & 30.09 & 1.36 \\
\hline 105 & Track 1 & $17.77 *$ & 27.27 & 0.05 & 0.02 & 20.28 & 1.64 \\
\hline 105 & Track 4 & $29.55^{*}$ & 26.43 & 0.05 & 0.02 & 31.96 & 1.53 \\
\hline 106 & Track 1 & 33.01 & $11.62 *$ & 0.01 & 0.01 & 34.85 & 0.75 \\
\hline 106 & Track 4 & 32.14 & $23.78 *$ & 0.03 & 0.02 & 34.17 & 1.53 \\
\hline 107 & Track 1 & $35.22 *$ & $13.93 *$ & 0.04 & 0.01 & 35.38 & 0.82 \\
\hline 107 & Track 4 & $28.21 *$ & $41.48 *$ & 0.23 & 0.03 & 32.89 & 1.56 \\
\hline 108 & Track 1 & $12.14 *$ & $19.63 *$ & 0.00 & 0.06 & 12.50 & 1.20 \\
\hline 108 & Track 4 & $26.57 *$ & $30.43 *$ & 0.10 & 0.03 & 28.86 & 1.63 \\
\hline
\end{tabular}

Significant differences between the attentional loads for each participant are highlighted with $*(p s<.01)$

Table 2 Mean error and variability in performance for the different conditions in Experiment 2 (all angles reported in degrees)

\begin{tabular}{|c|c|c|c|c|c|c|}
\hline \multirow[t]{2}{*}{ ID } & \multirow[t]{2}{*}{ Condition } & \multicolumn{5}{|c|}{ Mean error (standard deviation) } \\
\hline & & $6^{\circ}$ & $8^{\circ}$ & $10^{\circ}$ & $12^{\circ}$ & $14^{\circ}$ \\
\hline 201 & Track 1 & $2.29(9.46)$ & $2.89(10.34)$ & $3.70(14.14)$ & $4.89(14.08)$ & $8.84(17.02)$ \\
\hline 201 & Track 4 & $3.33(8.72)$ & $0.44(11.49)$ & $3.48(14.68)$ & $7.14(15.29)$ & $7.32(17.07)$ \\
\hline 202 & Track 1 & $1.81(16.00)$ & $5.11(20.00)$ & $6.92(22.51)$ & $6.30(22.19)$ & $10.35(23.69)$ \\
\hline 202 & Track 4 & $1.95(14.55)$ & $5.10(17.93)$ & $9.07(21.25)$ & $12.77(23.01)$ & $11.97(25.07)$ \\
\hline 203 & Track 1 & $3.97(12.08)$ & $3.80(14.04)$ & $5.56(11.80)$ & $9.35(14.67)$ & $9.63(\mathbf{1 2 . 3 5})$ \\
\hline 203 & Track 4 & $1.47(11.25)$ & $6.53(14.37)$ & $3.95(15.24)$ & $8.28(13.85)$ & $6.16(\mathbf{1 7 . 1 0})$ \\
\hline 204 & Track 1 & $-0.94 *(8.43)$ & $-1.35(12.86)$ & $1.43(15.13)$ & $4.56(16.33)$ & $3.22(19.47)$ \\
\hline 204 & Track 4 & $2.68 *(9.26)$ & $1.52(12.17)$ & $6.02(14.88)$ & $5.05(20.23)$ & $6.74(21.42)$ \\
\hline 205 & Track 1 & $1.78(12.73)$ & $2.83(17.45)$ & $5.65(19.96)$ & 8.56 (20.97) & $12.52(23.95)$ \\
\hline 205 & Track 4 & $2.84(12.92)$ & $3.59(18.56)$ & $11.03(20.02)$ & $10.85(24.83)$ & $12.21(26.39)$ \\
\hline 206 & Track 1 & $11.61(15.79)$ & $13.72(19.01)$ & $14.54(19.74)$ & $20.60(20.97)$ & $18.89(23.30)$ \\
\hline 206 & Track 4 & $10.74(16.10)$ & $12.76(16.59)$ & $18.42(18.13)$ & $20.74(19.63)$ & 21.09 (25.91) \\
\hline 207 & Track 1 & 3.27 (12.87) & $6.62(15.67)$ & $5.34(20.16)$ & $8.93(23.56)$ & $11.50(24.99)$ \\
\hline 207 & Track 4 & $2.45(13.85)$ & $3.18(15.67)$ & 7.63 (19.34) & $9.62(24.59)$ & $10.07(27.90)$ \\
\hline 208 & Track 1 & $0.96(15.09)$ & $-0.86(17.07)$ & $3.92(19.47)$ & $6.78(20.44)$ & $9.81(19.65)$ \\
\hline 208 & Track 4 & $3.91(12.06)$ & $3.18(15.60)$ & $9.93(17.53)$ & $10.56(17.76)$ & $11.42(19.96)$ \\
\hline
\end{tabular}

Significant differences between the attentional loads are highlighted in with *, and significant variance differences are in bold $(p \mathrm{~s}<.01)$ 


\section{References}

Alvarez, G. A., \& Franconeri, S. L. (2007). How many objects can you track? Evidence for a resource-limited attentive tracking mechanism. Journal of Vision, 7(13), 1-10. https://doi.org/10.1167/7.13. 14.

Awh, E., Armstrong, K. M., \& Moore, T. (2006). Visual and oculomotor selection: Links, causes and implications for spatial attention. Trends in Cognitive Sciences, 10(3), 124-130. https://doi.org/10.1016/j. tics.2006.01.001

Brainard, D. H. (1997). The Psychophysics Toolbox. Spatial Vision, 10(4), 433-436. https://doi.org/10.1163/156856897X00357

Casarotti, M., Lisi, M., Umiltà, C., \& Zorzi, M. (2012). Paying attention through eye movements: A computational investigation of the premotor theory of spatial attention. Journal of Cognitive Neuroscience, 24(7), 1519-1531. https://doi.org/10.1162/jocn_a 00231

Cavanagh, P., \& Alvarez, G. A. (2005). Tracking multiple targets with multifocal attention. Trends in Cognitive Sciences, 9(7), 349-354. https://doi.org/10.1016/j.tics.2005.05.009

Deubel, H., \& Schneider, W. X. (1996). Saccade target selection and object recognition: Evidence for a common attentional mechanism. Vision Research, 36(12), 1827-1837. https://doi.org/10.1016/00426989(95)00294-4

Drew, T., McCollough, A. W., Horowitz, T. S., \& Vogel, E. K. (2009). Attentional enhancement during multiple-object tracking. Psychonomic Bulletin \& Review, 16(2), 411-417. https://doi.org/ 10.3758/PBR.16.2.411

Fencsik, D. E., Klieger, S. B., \& Horowitz, T. S. (2007). The role of location and motion information in the tracking and recovery of moving objects. Perception \& Psychophysics, 69(4), 567-577. https://doi.org/10.3758/BF03193914

Horowitz, T. S., \& Cohen, M. A. (2010). Direction information in multiple object tracking is limited by a graded resource. Attention, Perception, \& Psychophysics, 72(7), 1765-1775. https://doi.org/ 10.3758/APP.72.7.1765

Keane, B. P., \& Pylyshyn, Z. W. (2006). Is motion extrapolation employed in multiple object tracking? Tracking as a low-level, non-predictive function. Cognitive Psychology, 52(4), 346-368. https://doi.org/10.1016/j.cogpsych.2005.12.001

Kwon, O.-S., Tadin, D., \& Knill, D. C. (2015). Unifying account of visual motion and position perception. Proceedings of the National Academy of Sciences, 112(26), 8142-8147. https://doi.org/10. 1073/pnas. 1500361112

Lisi, M., Bonato, M., \& Zorzi, M. (2015). Pupil dilation reveals top-down attentional load during spatial monitoring. Biological Psychology, 112, 39-45. https://doi.org/10.1016/j.biopsycho.2015.10.002

Lisi, M., \& Cavanagh, P. (2015). Dissociation between the perceptual and saccadic localization of moving objects. Current Biology, 25(19), 2535-2540. https://doi.org/10.1016/j.cub.2015.08.021

Lisi, M., \& Cavanagh, P. (2017). Different spatial representations guide eye and hand movements. Journal of Vision, 17(2), 12-12. https:// doi.org/10.1167/17.2.12

Meyerhoff, H. S., Papenmeier, F., \& Huff, M. (2017). Studying visual attention using the multiple object tracking paradigm: A tutorial review. Attention, Perception, \& Psychophysics, 79(5), 12551274. https://doi.org/10.3758/s13414-017-1338-1

Pylyshyn, Z. W. (1989). The role of location indexes in spatial perception: A sketch of the FINST spatial-index model. Cognition, 32(1), 6597. https://doi.org/10.1016/0010-0277(89)90014-0

Pylyshyn, Z. W. (2004). Some puzzling findings in multiple object tracking: I. Tracking without keeping track of object identities. Visual Cognition, 11(7), 801-822. https://doi.org/10.1080/ 13506280344000518

Rizzolatti, G., Riggio, L., Dascola, I., \& Umiltá, C. (1987). Reorienting attention across the horizontal and vertical meridians: evidence in favor of a premotor theory of attention. Neuropsychologia, 25(1A), 31-40. https://doi.org/10.1016/0028-3932(87)90041-8

Shapiro, A., Lu, Z.-L., Huang, C.-B., Knight, E., \& Ennis, R. (2010). Transitions between central and peripheral vision create spatial/ temporal distortions: A hypothesis concerning the perceived break of the curveball. PLOS ONE, 5(10), e13296. https://doi.org/10. 1371/journal.pone.0013296

Tse, P. U., \& Hsieh, P. J. (2006). The infinite regress illusion reveals faulty integration of local and global motion signals. Vision Research, 46(22), 3881-3885. https://doi.org/10.1016/j.visres.2006.06.010 